\title{
Risk factors and outcomes of tapering surgery for small intestinal dilatation in pediatric short bowel syndrome
}

\section{Hukkinen, Maria}

2017-07

Hukkinen, M , Kivisaari , R , Koivusalo , A \& Pakarinen , M P 2017 , ' Risk factors and outcomes of tapering surgery for small intestinal dilatation in pediatric short bowel syndrome ' , Journal of Pediatric Surgery , vol. 52 , no. 7 , pp. 1121-1127 . https://doi.org/10.1016/j.jpedsurg.2017.01.052

http://hdl.handle.net/10138/297801

https://doi.org/10.1016/j.jpedsurg.2017.01.052

publishedVersion

Downloaded from Helda, University of Helsinki institutional repository.

This is an electronic reprint of the original article.

This reprint may differ from the original in pagination and typographic detail.

Please cite the original version. 


\title{
Risk factors and outcomes of tapering surgery for small intestinal dilatation in pediatric short bowel syndrome
}

\author{
Maria Hukkinen ${ }^{\mathrm{a}, \mathrm{b}}$, Reetta Kivisaari ${ }^{\mathrm{c}}$, Antti Koivusalo ${ }^{\mathrm{a}, \mathrm{b}}$, Mikko P. Pakarinen ${ }^{\mathrm{a}, \mathrm{b}, *}$ \\ a Pediatric Liver and Gut Research Group, Children's Hospital, Helsinki University Hospital, University of Helsinki, Helsinki, Finland \\ b Section of Pediatric Surgery, Children's Hospital, Helsinki University Hospital, University of Helsinki, Helsinki, Finland \\ c HUS Medical Imaging Center, Children's Hospital, Helsinki University Hospital, University of Helsinki, Helsinki, Finland
}

\section{A R T I C L E I N F O}

\section{Article history:}

Received 19 June 2016

Received in revised form 20 November 2016

Accepted 24 January 2017

\section{Key words:}

Short bowel syndrome

Intestinal failure

Tapering surgery

Serial transverse enteroplasty

Longitudinal intestinal lengthening and tapering

\section{A B S T R A C T}

Background: In remains unclear why in some short bowel syndrome (SBS) patients, the remaining small bowel (SB) dilates excessively leading to requirement of tapering surgery.

Methods: Among SBS children, we retrospectively analyzed risk factors for tapering surgery with logistic regression and compared the outcome of operated patients $(n=16)$ to those managed conservatively $(n=44)$ with Cox proportional hazards regression.

Results: SBS was caused by necrotizing enterocolitis (NEC) $(n=31)$, SB atresia (SBA) $(n=13)$, midgut volvulus $(n=12)$, or gastroschisis $(n=4)$. Patients with spontaneous symptomatic SB dilatation unable to wean parenteral nutrition (PN) underwent tapering surgery at median age of 1.04 (interquartile range 0.70-3.27) years. Missing ICV was related to an 8-fold $(p=0.003)$ increased risk while SBA diagnosis was related to a 13-fold risk of tapering surgery $(p<0.001)$. Increasing SB length and NEC diagnosis were protective of tapering $(p=$ 0.027-0.004). Of operated patients, 75\% reached enteral autonomy during follow-up and their postoperative adjusted PN weaning rate was similar to nonoperated children $(p=0.842)$.

Conclusion: SBS children with short remaining SB, missing ICV, and SBA etiology are more likely while NEC patients are less likely than others to necessitate tapering surgery. Postoperative PN weaning rates were comparable to patients who initially had more favorable intestinal anatomy and adapted without surgery.

(c) 2017 Elsevier Inc. All rights reserved.
Intestinal failure (IF) in children is defined as reduction of functional intestinal mass below the minimum needed for sufficient absorption of fluid and nutrients, and requirement of long-term parenteral nutrition (PN) to sustain survival and maintain adequate growth instead [1,2]. The most common reason for IF among children is short bowel syndrome (SBS) caused by extensive small bowel (SB) resection, indicated during infancy for conditions such as necrotizing enterocolitis (NEC), midgut volvulus (MGV), SB atresia (SBA), and gastroschisis [3,4]. Increasing remaining bowel length, preserved ileocecal valve (ICV), and reestablishment of intestinal continuity improve the chances for weaning off $\mathrm{PN}$, which is the main goal in the treatment of SBS patients $[2,3,5,6]$.

After resection, the remaining SB undergoes structural and functional changes to improve its absorptive capacity $[7,8]$. In some patients, the remaining SB dilates as part of the physiological adaptation process

Abbreviations: ALT, alanine aminotransferase; BO, bacterial overgrowth; HR, hazard ratio; ICV, ileocecal valve; IF, intestinal failure; IQR, interquartile range; LILT, longitudinal intestinal lengthening and tapering; MGV, midgut volvulus; NEC, necrotizing enterocolitis; OR, odds ratio; PN, parenteral nutrition; SB, small bowel; SBA, SB atresia; STEP, serial transverse enteroplasty; $\mathrm{CI}$, confidence interval.

* Corresponding author at: Children's Hospital, Helsinki University Hospital, P.O. Box 281, 00029 HUS, Helsinki, Finland. Tel.: +358 50 4272981; fax: + 358947175737.

E-mail address: mikko.pakarinen@hus.fi (M.P. Pakarinen).
$[3,7,8]$. Although this increases the bowel absorptive surface, excessive SB dilatation may also impair intestinal motility and promote bacterial overgrowth (BO), which in turn predispose SBS patients to septic infections, D-lactate acidosis, and malabsorption $[9,10]$. We have recently demonstrated that adaptive SB dilatation is an independent risk factor for prolonged PN duration and decreased survival in SBS children [11]. In addition to PN dependence, anatomy of the remaining bowel and the etiology of SBS possibly influence the risk of excessive adaptive dilatation [10-13].

Tapering surgery of dilated SB in order to relieve the related functional problems has become increasingly popular during recent years especially in pediatric patients [14-20]. In serial transverse enteroplasty (STEP) and longitudinal intestinal lengthening and tapering (LILT), SB length is increased by narrowing the dilated intestine while preserving all absorptive mucosal surface $[19,21]$. Alternatively, dilated parts may be narrowed by simple tapering enteroplasty or segmental resection when the remaining bowel is not critically short [22]. SBS patients unable to wean from PN because of complications related to SB dilatation are considered as candidates for tapering surgery $[15,17,19]$. Among such patients, tapering increases the likelihood of weaning off PN possibly by reducing BO, by improving bowel motility, and by promoting absorption [14-20,23]. Tapering may also ameliorate liver dysfunction by decreasing the need for PN [24]. However, few studies have compared 
the outcomes of SBS patients having undergone tapering to those treated without surgery [25], and it remains unclear how SBS children necessitating tapering differ from patients who adapt without surgical procedures.

Identification of risk factors for the need of tapering surgery would help to recognize the most susceptible SBS patients earlier and target particular attention at them. Moreover, as tapering surgery has become an established practice in the treatment of SBS, its efficacy should be evaluated not only by comparing the preoperative and postoperative results but also by paralleling the outcome of operated patients to those managed conservatively. To this end, we aimed to analyze risk factors for the need of tapering surgery for spontaneous adaptation-related excessive small intestinal dilatation, as well as to study the respective PN weaning and survival rates in SBS children treated with or without tapering surgery.

\section{Methods}

\subsection{Patients}

This was a retrospective study including all patients with IF caused by SBS treated by our intestinal rehabilitation program [26] in $2002-2015(n=61)$. Inclusion criteria were SB resection $>50 \%$ of ageadjusted reference value, or duration of $\mathrm{PN}$ for over two consecutive months $[4,27,28]$. Patients with tapering surgery following controlled bowel expansion $(n=1)$ and other etiology of IF ( $n=33$ ), such as primary motility disorders, mucosal diseases, and lymphatic disorders were excluded. Medical records were reviewed for SBS etiology, gestational age and weight, duration of PN, plasma citrulline levels, intestinal anatomy, and SB width in contrast series. Bowel length was expressed both as absolute values and as percentage of age-adjusted reference values [28] while the presence of ICV and ileum was recorded as absent or present. Maximal width of SB perpendicular to the longitudinal axis of the bowel was measured in contrast SB series as described previously [11]. SB width was expressed both as $\mathrm{mm}$ (absolute SB width) and as internally standardized ratio to the height of the fifth lumbar vertebra (SB diameter ratio) [11]. At last follow-up, plasma alanine aminotransferase (ALT) and bilirubin levels as well as liver biopsy findings were recorded, and growth was assessed by using height $z$-scores as well as ageadjusted ISO-BMI for children aged 2-18 years; age-adjusted weightto-height percentiles (based on national data) for children under 2 years of age; and the BMI for patients older than 18 years [26].

\subsection{Tapering surgery}

Tapering surgery was considered in SBS patients with excessive bowel dilatation who were unable to wean from parenteral support and presented with complications related to symptoms of SB dilatation, such as BO or dysmotility. The presence of one or several dilated SB segments clearly exceeding the diameter of normal-caliber SB in the same contrast SB series was a prerequisite for tapering surgery. BO and dysmotility were diagnosed based on clinical symptoms, including abdominal bloating, vomiting, increased intestinal secretions, and inability to advance enteral nutrition. Altogether 16 patients underwent tapering procedures, including STEP, LILT, tapering enteroplasty, and resection of dilated bowel segment. In this study, resection of dilated segment was included in tapering procedures because it was used as STEP, LILT, or tapering enteroplasty to treat excessively dilated SB, however, for patients with limited dilatation and sufficient remaining small intestinal length. Previously standardized surgical techniques for STEP and LILT were followed $[17,19]$. Simple tapering of a dilated bowel segment was performed with a linear stapler along the long axis of a dilated bowel segment [22]. Indications for tapering surgery and possible postoperative complications were recorded. Primary and retapering procedures were analyzed separately. One of the primary tapering procedures (STEP) had been performed in another hospital, after which the patient was referred to us and later underwent a reoperation for symptomatic redilatation.

\subsection{Statistical methods}

Data are presented as medians with interquartile ranges (IQR) or as frequencies. Spearman rank correlation was used to examine associations between variables. Mann-Whitney $U$ test was used to compare continuous variables, Wilcoxon signed-rank test to compare repeated measurements within groups, and Fisher exact test to compare frequencies between subgroups. We conducted simple and multiple logistic regression analyses to further estimate the magnitude of association between different variables and the need for tapering surgery. The odds ratios (ORs) with 95\% confidence intervals (CIs) were computed, and statistically significant predictors from the simple regression model were chosen for the multiple regression analysis. As SB dilatation was a prerequisite for tapering surgery, SB width or SB diameter ratio were not included in these analyses. Kaplan-Meier plots with the logrank test were used to compare the PN weaning rates of operated patients to nonoperated patients, and those receiving PN at the end of follow-up or who died while receiving PN were censored from the analysis. Finally, we studied the predictive values for different variables on cumulative rates of weaning off PN and survival by generating hazard ratios (HRs) with 95\% CIs with Cox proportional hazards regression model. Variables showing statistically significant univariate associations with PN weaning rates were included in the multivariate model. The level of significance was set at $p<0.05$ and all analyses were carried out with SPSS version 22 .

\subsection{Ethics}

The study protocol was approved by the hospital ethical committee.

\section{Results}

\subsection{Patient characteristics}

We identified 60 eligible patients with IF caused by SBS (males 58\%, $n=35$ ). At the time of study, intestinal continuity had been reestablished in all and none had an enterostomy. Baseline diagnoses were necrotizing enterocolitis (NEC) $(n=31)$, midgut volvulus $(\mathrm{MGV})(n=12)$, small bowel atresia (SBA) $(n=13)$, and gastroschisis $(n=4)$ (Table 1$)$. Four patients with both SBA and gastroschisis were analyzed in the SBA group. Median residual small bowel length was 40 (25-60) cm while colon length was $32(24-41) \mathrm{cm}$, corresponding to $25 \%(17 \%-44 \%)$ and $93 \%(61 \%-100 \%)$ of age-adjusted reference values, respectively [28]. The ICV was missing in 28 (47\%) and no ileum was remaining in 27 (45\%). Median gestational age was 34 (28-36) weeks and birth weight was 2090 (960-3035) grams. Median plasma citrulline was $13(10-17)$ before and $24(20-29)$ after weaning off PN $(p<0.001)$.

\subsection{Tapering surgery}

Altogether 16 patients (27\%) had undergone tapering procedures. In addition to bowel dysmotility, inability to increase enteral intake and BO were the most common indications for tapering surgery (Table 2). The patient having undergone bowel resection had been previously off PN before developing symptomatic segmental SB dilatation and becoming PN dependent again. Postoperative complications requiring relaparotomy occurred in two while milder complications in two patients (Table 2). Preoperative contrast SB series were performed on 10 patients while postoperative contrast SB series on 13 patients. The maximal SB diameter ratio in these contrast series decreased significantly following tapering (Table $2, p=0.009$ ), while no decrease in maximal absolute SB width was observed. 
Table 1

Characteristics of patients managed with $(n=16)$ or without $(n=44)$ tapering surgery.

\begin{tabular}{|c|c|c|c|}
\hline & Tapering $(n=16)$ & No tapering $(n=44)$ & $p$-Value \\
\hline \multicolumn{4}{|l|}{ Baseline diagnosis } \\
\hline NEC, $n(\%)$ & $3(19 \%)$ & $28(64 \%)$ & 0.003 \\
\hline SBA, $n(\%)$ & $9(56 \%)$ & $4(9 \%)$ & $<0.001$ \\
\hline MGV, $n(\%)$ & $3(19 \%)$ & $9(20 \%)$ & 1.000 \\
\hline Gastroschisis, $n(\%)$ & $1(6 \%)$ & $3(7 \%)$ & 1.000 \\
\hline Remaining SB length (cm) & $30(23-41)$ & $50(30-61)$ & 0.019 \\
\hline Remaining SB length (\%) & $24(18-26)$ & $26(17-49)$ & 0.270 \\
\hline Remaining colon length $(\mathrm{cm})$ & $28(21-31)$ & $39(24-55)$ & 0.026 \\
\hline Remaining colon length (\%) & $75(59-90)$ & $100(66-100)$ & 0.061 \\
\hline ICV missing, $n(\%)$ & $13(81 \%)$ & $15(34 \%)$ & 0.003 \\
\hline Maximal SB width (mm) & $41(36-60)$ & $25(20-30)$ & $<0.001$ \\
\hline Maximal SB diameter ratio & $3.54(2.20-4.00)$ & $2.74(2.08-3.17)$ & 0.064 \\
\hline Total duration of PN (months) & $19.5(10.0-47.6)$ & $6.72(3.05-12.5)$ & $<0.001$ \\
\hline Birth weight (grams) & $2400(1610-3510)$ & $1250(740-2400)$ & 0.030 \\
\hline Gestational age (weeks) & $35(31-36)$ & $30(25-35)$ & 0.140 \\
\hline Weaned off PN, $n(\%)$ & $12(75 \%)$ & $38(86 \%)$ & 0.433 \\
\hline Survival, $n(\%)$ & $15(94 \%)$ & $40(91 \%)$ & 1.000 \\
\hline Follow-up plasma ALT & $31(21-46)$ & $27(19-46)$ & 0.543 \\
\hline Follow-up plasma bilirubin & $6(3-8)$ & $6(3-11)$ & 0.467 \\
\hline Follow-up ISO-BMI $(n=42)$ & $20.6(17.8-24.2)$ & $19.8(18.3-21.8)$ & 0.990 \\
\hline Follow-up BMI $(n=6)$ & $17.5(17.2-17.7)$ & $16.9(16.4-19.0)$ & 0.700 \\
\hline Follow-up weight-for-height $(\%)(n=12)$ & $-7.0(-7.5$ to -2.0$)$ & $-7.0(-7.0$ to -4.0$)$ & 1.000 \\
\hline Follow-up height z-score $(n=54)$ & $-0.95(-2.4$ to 0.00$)$ & $-1.2(-1.8$ to -0.70$)$ & 0.743 \\
\hline
\end{tabular}

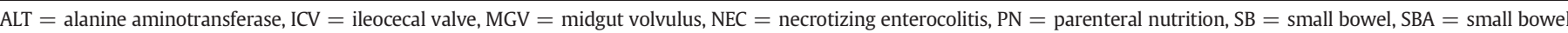

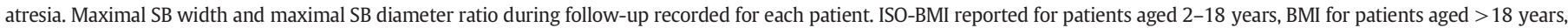
and weight-for-height percentiles for patients aged $<2$ years at last follow-up. Height z-scores reported for all patients younger than 18 years.

\subsection{Predictors of tapering surgery}

Patients who underwent tapering had higher maximal SB width, shorter remaining SB and colon, higher birth weight, and ICV missing more frequently when compared to patients not undergoing surgery (Table 1). SBA patients were more likely while NEC patients less likely to undergo tapering than patients with MGV or gastroschisis. According to univariate regression, missing ICV, decreasing remaining SB length,

Table 2

Operative characteristics of patients undergoing tapering surgery $(n=16)$.

\begin{tabular}{|c|c|}
\hline Tapering procedure, total $n$ & 16 \\
\hline STEP, $n(\%)^{*}$ & $10(63 \%)$ \\
\hline LILT, $n(\%)$ & $2(13 \%)$ \\
\hline Tapering enteroplasty, $n(\%)$ & $3(19 \%)$ \\
\hline Resection, $n(\%)$ & $1(6 \%)$ \\
\hline Indications for surgery ${ }^{* *}$, total $n$ & 15 \\
\hline Dysmotility, $n(\%)$ & $15(100 \%)$ \\
\hline Bacterial overgrowth, $n(\%)$ & $10(67 \%)$ \\
\hline Inability to increase enteral intake, $n(\%)$ & $12(80 \%)$ \\
\hline Vomiting, $n(\%)$ & $8(53 \%)$ \\
\hline D-lactate acidosis, $n(\%)$ & $2(13 \%)$ \\
\hline Obstructive symptoms, $n(\%)$ & $2(13 \%)$ \\
\hline Age at tapering (years) $)^{* *}$ & $1.04(0.70-3.27)$ \\
\hline Postoperative complications ${ }^{* *}$, total $n$ & $4(27 \%)$ \\
\hline Wound infection, $n(\%)$ & $1(7 \%)$ \\
\hline Intraabdominal hematoma, $n(\%)$ & $1(7 \%)$ \\
\hline Wound dehiscence, $n(\%)$ & $1(7 \%)$ \\
\hline Bowel perforation, $n(\%)$ & $1(7 \%)$ \\
\hline Retapering, $n(\%)$ & $6(38 \%)$ \\
\hline Weaned off PN, $n(\%)$ & $12(75 \%)$ \\
\hline Time to wean off PN after tapering (months) & $5.6(0.30-20.3)$ \\
\hline Preoperative NG/gastrostomy tube feeding & $6(38 \%)$ \\
\hline Postoperative NG/gastrostomy tube feeding & $10(63 \%)$ \\
\hline Preoperative maximal SB diameter ratio & $3.55(2.19-3.83)$ \\
\hline Postoperative maximal SB diameter ratio & $2.32(1.92-2.87)$ \\
\hline Preoperative maximal SB width (mm) & $36(33-40)$ \\
\hline Postoperative maximal SB width (mm) & $35(29-60)$ \\
\hline
\end{tabular}

Preoperative maximal small bowel (SB) width and SB diameter ratio measurements available for 10 patients, postoperative measurements available for 13 patients.

LILT = longitudinal intestinal lengthening and tapering, NG = nasogastric, $\mathrm{PN}=$ parenteral nutrition, $\mathrm{STEP}=$ serial transverse enteroplasty .

* Including one STEP procedure performed in another hospital.

** Data missing for the patient who underwent primary surgery in another hospital. and SBA etiology compared to MGV and gastroschisis were significant predictors of surgery (Table 3 ). In the multiple regression analysis adjusted for these statistically significant variables, remaining SB length (OR 0.96, 95\% CI 0.92-1.00, $p=0.050$ ) and NEC etiology compared to gastroschisis and MGV (OR 0.02, 95\% CI 0.00-0.45, $p=0.021$ ) remained statistically significant predictors of tapering surgery. When all other patients were compared to NEC, their univariate OR for tapering was 7.58 (95\% CI 1.88-30.7, $p=0.004$ ), suggesting that NEC etiology was protective of tapering surgery. Comparing SBA patients to others, their OR for surgery was 12.9 (95\% CI 3.09-53.5, $p<0.001$ ).

\subsection{Retapering surgery}

Retapering was performed in six patients (NEC, $n=3$; SBA, $n=2$; MGV, $n=1)$ at the age of $2.78(1.73-14.8)$ years, 1.34 (0.96-17.0) years after initial tapering. Reoperations included four STEP procedures, one bowel resection, and one tapering enteroplasty. In addition to dysmotility $(n=6)$, BO $(n=4)$, inability to increase enteral intake $(n=4)$, obstructive symptoms $(n=2)$, and weight loss $(n=1)$ were among indications for retapering. In addition, one patient underwent

Table 3

Associations between the need for tapering surgery and baseline patient characteristics assessed with simple logistic regression.

\begin{tabular}{lll}
\hline & OR $(95 \% \mathrm{CI})$ & $p$-Value \\
\hline Etiology & & \\
$\quad$ Gastroschisis/MGV & 1.00 (Ref) & \\
NEC & $0.32(0.06-1.66)$ & 0.176 \\
SBA & $6.75(1.32-34.57)$ & 0.022 \\
Remaining SB length (cm) & $0.97(0.94-1.00)$ & 0.027 \\
Remaining SB length (\%) & $0.97(0.94-1.01)$ & 0.797 \\
Remaining colon length (\%) & $0.99(0.96-1.01)$ & 0.231 \\
ICV missing & $8.38(2.06-34.03)$ & 0.003 \\
PN duration & $1.01(0.98-1.04)$ & 0.562 \\
Birth weight & $1.00(-)$ & 0.100 \\
Gestational age & $1.21(0.96-1.52)$ & 0.110
\end{tabular}

Duration of parenteral nutrition (PN) at tapering analyzed for operated patients and total PN duration for others.

$95 \% \mathrm{CI}=95 \%$ confidence interval, $\mathrm{ICV}=$ ileocecal valve, $\mathrm{MGV}=$ midgut volvulus, $\mathrm{NEC}=$ necrotizing enterocolitis, $\mathrm{OR}=$ odds ratio, $\mathrm{SBA}=$ small bowel atresia 
STEP as a third tapering procedure. No postoperative complications after retapering were recorded. Patients' median SB length was 42 (25-28) cm, corresponding to $24 \%(22 \%-29 \%)$ of age-adjusted bowel length, and none had ICV remaining; however, no statistically significant differences in patient characteristics, PN duration, or bowel anatomy were observed between patients undergoing retapering vs. only one tapering procedure. Maximal absolute SB width was $50(25-88) \mathrm{mm}$ prior to retapering and $28(25-34) \mathrm{mm}$ postoperatively, and maximal SB diameter ratio was 2.26 (1.92-4.00) preoperatively, decreasing to 2.04 (1.93-2.45). No statistically significant decrease in either measure, however, was observed.

\subsection{Predictors of weaning off parenteral nutrition}

Of patients undergoing tapering, $88 \%(n=14)$ were continuously PN dependent at time of surgery while $25 \%(n=4)$ required any kind of parenteral support at the end of the follow-up ( $p=0.001)$. Although two patients were not on continuous PN at time of tapering, they had experienced repeated episodes of dehydration necessitating parenteral rehydration. Preoperative duration of PN was 10.5 (7.65-14.3) months, postoperative PN duration was 8.17 (1.09-28.2) months, and total PN duration was longer in operated patients when compared to others (Table 1$)$. Of patients treated without surgery, $14 \%(n=6)$ were still PN dependent or had died while on PN by the end of the follow-up ( $p=0.433$ when compared to operated patients). Although the overall cumulative probability of weaning off PN was higher in patients who were managed without tapering surgery (Table 4, Figs. 1A and 2A), the cumulative PN weaning rates analyzed after tapering surgery were comparable between subgroups (Figs. 1B and 2B).

\subsection{Survival, liver function, and growth}

Survival was comparable between operated patients and those treated without surgery (Table 1), and none of the patients in either group had been listed for intestinal transplantation by the end of the follow-up. No significant predictors of survival were identified; the HRs for the length or percentage of remaining SB or colon, the presence of ICV, maximal SB width or maximal SB ratio, gestational age, birth weight, tapering surgery, and etiology of SBS were nonsignificant. At last follow-up at a median age of 4.99 (2.83-10.23) years, plasma bilirubin and ALT, height $z$-scores, and weight parameters in children (ISOBMI for those aged 2-18 years; weight-for-height percentiles for those younger than 2 years) were in normal range, while BMI of patients older than 18 years $(n=6)$ was slightly below normal (Table 1 ). Follow-up liver biopsies (total $n=39$; operated patients $n=14$ ) showed cholestasis in 9 (23\%), steatosis in 9 (23\%), and any fibrosis in $24(62 \%)$ patients, of which Metavir stage 1 was observed in 12 (31\%), stage 2 in 11 (28\%), and stage 3 in one patient (5\%). The presence of histological cholestasis or the severity of fibrosis did not differ between patient subgroups, whereas steatosis was more frequent among operated patients than others ( 8 vs. $1, p<0.001$ ).

\section{Discussion}

This study demonstrates that in SBS children, short remaining SB, missing ICV, and baseline diagnosis of SBA are associated with an increased need for tapering surgery, while NEC patients are more likely than others to adapt without surgery. Total duration of PN was longer in children who underwent tapering surgery, however, by the end of the follow-up, PN weaning and survival rates were similar among operated and nonoperated patients. After tapering surgery, 75\% of children were able to reach enteral autonomy, and the cumulative PN weaning rates were comparable to patients treated without adaptive surgery who had a significantly longer intestinal remnant and more frequently preserved ICV.

The benefits of tapering surgery in PN dependent SBS patients are well-documented [14-20,24]. In most cohorts, median SB length of operated patients has been $<50 \mathrm{~cm}$, most have had no ICV remaining, and gastroschisis and SBA have been reported as predominant diagnoses over NEC and MGV $[14,15,18,20,25]$. PN dependence is an established risk factor for BO and SB dilatation [9,10,12,13,29], in addition, we have recently demonstrated that SBS children with short remaining SB and missing ICV have wider SB than others, and SBA compared to other etiologies relates to an increased SB diameter ratio [11]. However, whether specific risk factors or certain patient characteristics relate to the need for tapering surgery among SBS patients has not been studied earlier. Despite some limitations, including the retrospective study design and relatively small patient sample, this is the first study systematically analyzing the predictors of tapering surgery and comparing the outcomes of SBS patients treated either with or without tapering. In addition to other tapering procedures, we included two resections of dilated SB segment in this study, since among children with sufficient bowel length, the end-result and objective of resection essentially correspond to those of STEP, LILT, and simple tapering.

Our results suggest that SBA patients are 13-fold more likely while NEC patients 8-fold less likely than others to require tapering. In SBA, smooth muscle hypertrophy as well as hypoplasia of intramural nerves and Cajal cells in the proximal, dilated bowel predispose to dysmotility [30-32]. The SB proximal to atresia dilates slowly during fetal development $[31,32]$ whereas in NEC, an ischemic injury develops during days in a previously intact bowel wall in usually markedly premature infants with a great potential to age-related bowel growth $[28,33]$. These distinct etiologies may explain the observed differences in the need for tapering surgery between SBS subgroups. Dysmotility is common also in gastroschisis, where the exposure of intestines to amniotic fluid, formation of inflammatory peel, and intramural histopathology including

Table 4

Hazard ratios (HR) with 95\% confidence intervals (CI) for weaning off parenteral nutrition.

\begin{tabular}{|c|c|c|c|c|}
\hline & \multicolumn{2}{|l|}{ Univariate } & \multicolumn{2}{|l|}{ Multivariate } \\
\hline & $\mathrm{HR}(95 \% \mathrm{CI})$ & p-value & $\mathrm{HR}(95 \% \mathrm{CI})$ & p-value \\
\hline Other etiology vs. SBA & $1.91(0.89-4.09)$ & 0.096 & & \\
\hline NEC vs. other etiology & $0.64(0.36-1.13)$ & 0.123 & & \\
\hline No tapering vs. tapering surgery & $2.75(1.36-5.57)$ & 0.005 & $2.35(1.11-5.00)$ & 0.026 \\
\hline Remaining SB length (\%) & $1.03(1.02-1.05)$ & $<0.001$ & $1.03(1.02-1.05)$ & $<0.001$ \\
\hline Remaining SB length (cm) & $1.03(1.02-1.04)$ & $<0.001$ & & \\
\hline Remaining colon length (\%) & $1.01(0.99-1.02$ & 0.424 & & \\
\hline ICV preserved & $1.79(1.01-3.17)$ & 0.046 & $1.37(0.73-2.58)$ & 0.333 \\
\hline Maximal SB diameter ratio & $0.75(0.52-1.08)$ & 0.117 & & \\
\hline Maximal SB width & $0.63(0.46-0.86)$ & 0.004 & & \\
\hline Gestational age & $0.94(0.86-1.03)$ & 0.170 & & \\
\hline Birth weight & $1.00(-)$ & 0.353 & & \\
\hline
\end{tabular}

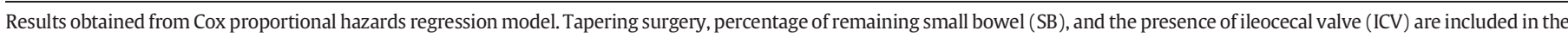
multivariate model. Maximal SB width was omitted from the multivariate analysis because of its high correlation with tapering surgery $(r=0.642)$. Total $n=60$.

$\mathrm{NEC}=$ necrotizing enterocolitis, $\mathrm{SBA}=$ small bowel atresia. 
A

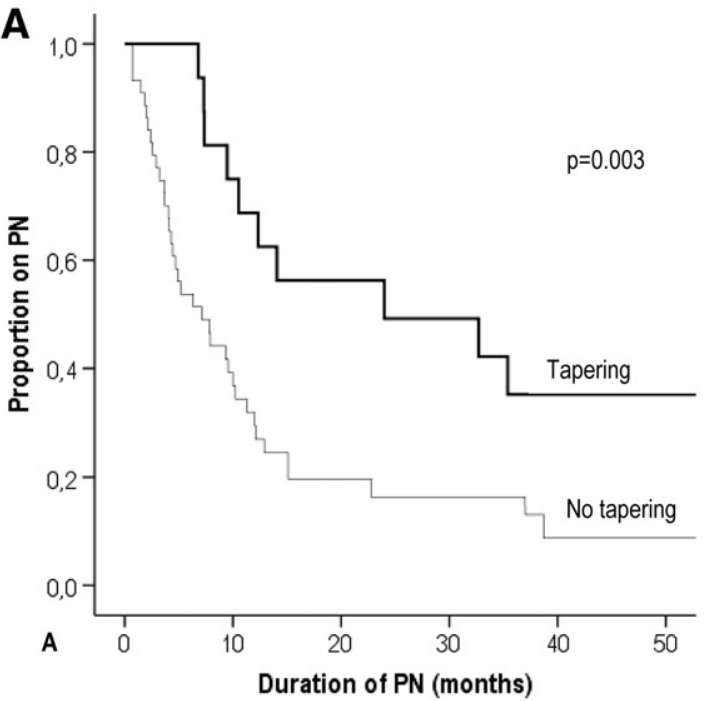

No. at risk

$\begin{array}{lllllll}\text { Tapering } & 16 & 12 & 8 & 7 & 5 & 4 \\ \text { No tapering } & 44 & 15 & 6 & 5 & 2 & 2\end{array}$

B

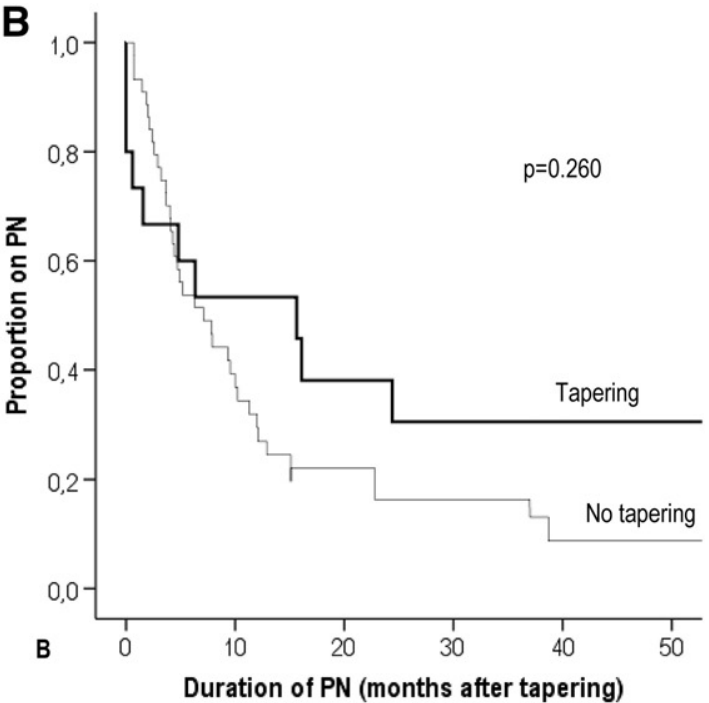

No. at risk

\begin{tabular}{|c|c|c|c|c|}
\hline Tapering & 16 & 7 & 5 & \\
\hline & 44 & 15 & 6 & \\
\hline
\end{tabular}

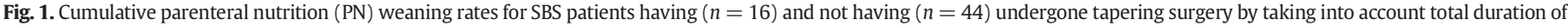
PN (A) and PN duration after tapering for operated patients (B). Survival analyzed with the Kaplan-Meier method and subgroups compared by using the log-rank test.

smooth muscle hyperplasia and decreased number of Cajal cells are considered responsible for abnormal motility [33-38]. Possibly because of small number of children with isolated gastroschisis, they showed no increased risk for tapering in the present study. However, as gastroschisis patients are overrepresented in other tapering reports $[14,18,20,25]$, active screening for SB dilatation in PN dependent patients with SBA, gastroschisis, or both seems justified.

Short length of remaining SB and missing ICV are known to relate to prolonged PN duration and poor prognosis among SBS patients $[3,5,19,39-41]$. This study demonstrates these anatomical factors are also independent risk factors for tapering surgery. It appears rational that patients with the shortest SB have the greatest tendency for bowel dilatation to increase its absorptive surface. The mechanism through which resection of the ileocecal region could promote dilatation and dysmotility is not as straightforward. Instead of the ICV acting as a physiological barrier prohibiting bacterial translocation from the colon, the distal ileum has been suggested to be more important in preventing SBS patients from the development of dysmotility, BO, and pathological dilatation $[10,12,13,42]$. Resection of the ileocecal region not only predisposes to bile acid malabsorption and altered intestinal microbiota, but may also weaken the propulsive peristalsis and interfere with regulation of adaptive bowel growth by impairing secretion of gut hormones which expression is confined to the distal ileum and the proximal colon, such as glucagon-like-peptides 1 and 2 and peptide YY $[8-10,43]$. As the remaining SB is not capable of fully compensating for these specific functions [8], removal of the ileum and proximal colon may be the reason for the increased need for tapering in patients with missing ICV. In the multiple regression analysis, we found missing ICV as the most significant risk factor for tapering. Moreover, none of the patients undergoing retapering had ICV remaining, further supporting the theory that its absence predisposes SBS children to complications related to SB dilatation.
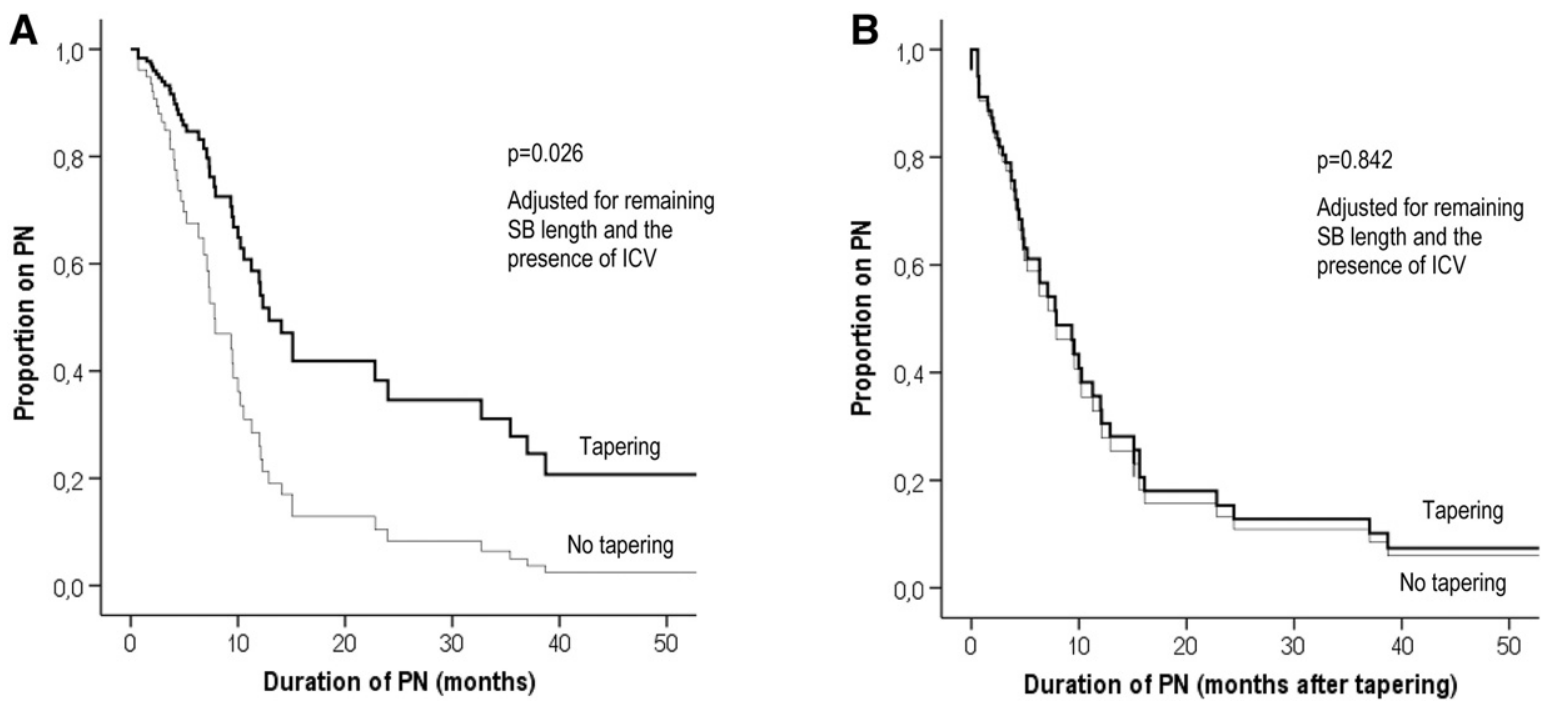

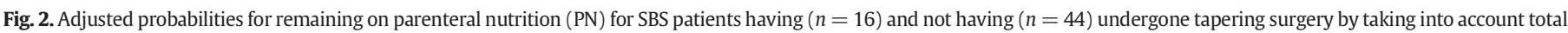
duration of PN (A) and PN duration after tapering for operated patients (B). Results obtained from the Cox proportional hazards regression analysis. 
As expected, short length of remaining SB and missing ICV were found to be prognostic of prolonged PN duration. In addition, patients having undergone tapering and those with wide SB in contrast series were less likely to wean off PN than others. In a previous study based on the same population, we have demonstrated that SB dilatation is a major risk factor for prolonged PN and decreased survival [11]. Here, the observed weaker association between SB dilatation and PN dependence is attributable to the main outcome being PN status at the end of the follow-up, when tapering surgery had partly overcome the negative predictive effect of dilatation. The finding that patients who underwent tapering had overall lower cumulative PN weaning rates than those treated conservatively likely reflects their less favorable intestinal anatomy, as SB length, width, and missing ICV were all predictive of both tapering surgery and weaning off PN. Importantly, after tapering, cumulative PN weaning rates were similar between subgroups, and the observed reduction in the proportion PN dependent patients postoperatively compares favorably with other reports $[14,16,18,20]$. Our results demonstrate that not only was surgery beneficial but it improved the outcome of operated patients to a level comparable to children who initially had longer SB, longer colon, and ICV preserved more frequently.

Our findings support the concept that tapering surgery should be considered in SBS children with excessively dilated SB when PN weaning process stagnates despite optimal medical and nutritional management $[15,17,19]$. Although postoperative complications are relatively uncommon, their possibility should be taken into account in the risk-benefit analysis of an individual patient. Considering all tapering and retapering procedures performed on our patients, the rate of postoperative complications was $17 \%$, which is comparable to other published reports $[15,18,44]$. After tapering, SB diameter ratio but not absolute SB width decreased significantly, possibly because of increasing age of patients between the measurements. As absolute SB width depends on patient age and size, SB diameter standardized to vertebral height is possibly a more reliable way to assess SB dilatation in children [11]. Unfortunately, a significant proportion of SBS patients develop redilatation and some also require retapering surgery [14,15,18,29]. While we were not able to identify any risk factors for retapering, the short length of remaining SB and absence of ICV in all patients undergoing retapering suggest that these anatomical characteristics continue to promote SB dilatation. Little evidence is available on the pathogenesis of redilatation $[9,10,29]$. Although postponing tapering surgery beyond the most efficient period of intestinal adaptation, first year after resection, might decrease the magnitude of redilatation, it may not be possible in all SBS children [15,19].

We have demonstrated that SBS children with resected ICV, short remaining SB, and SBA diagnosis are likely to require tapering surgery to facilitate weaning off PN, while NEC patients more probably than others adapt without surgery. In addition to carefully monitoring SBS children with poor prognostic characteristics; short remaining SB and missing ICV, attention should be drawn to SBS etiology which influences the need for tapering surgery. The probability of weaning off PN after tapering was comparable to children who required no adaptive surgery, demonstrating that in carefully selected patients, tapering surgery promotes enteral autonomy efficiently.

\section{Financial support}

This study was supported by research grants from the Sigrid Jusélius Foundation, the Finnish Pediatric Research Foundation, and the Helsinki University Central Hospital Grant.

\section{References}

[1] Goulet O, Olieman J, Ksiazyk J, et al. Neonatal short bowel syndrome as a model of intestinal failure: physiological background for enteral feeding. Clin Nutr 2013;32: $162-71$.
[2] Squires RH, Duggan C, Teitelbaum DH, et al. Natural history of pediatric intestinal failure: initial report from the pediatric intestinal failure consortium. J Pediatr 2012; $161: 723-8 . e 2$

[3] Amin SC, Pappas C, Iyengar H, et al. Short bowel syndrome in the NICU. Clin Perinatol 2013;40:53-68.

[4] D'Antiga L, Goulet O. Intestinal failure in children: the European view. J Pediatr Gastroenterol Nutr 2013;56:118-26.

[5] Quiros-Tejeira RE, Ament ME, Reyen L, et al. Long-term parenteral nutritional support and intestinal adaptation in children with short bowel syndrome: a 25-year experience. J Pediatr 2004;145:157-63.

[6] Sudan D, Thompson J, Botha J, et al. Comparison of intestinal lengthening procedures for patients with short bowel syndrome. Ann Surg 2007;246:593-601 [discussion 601-4].

[7] McDuffie LA, Bucher BT, Erwin CR, et al. Intestinal adaptation after small bowel resection in human infants. J Pediatr Surg 2011;46:1045-51.

[8] Tappenden KA. Intestinal adaptation following resection. JPEN J Parenter Enteral Nutr 2014;38:S23-31.

[9] Quigley EM, Quera R. Small intestinal bacterial overgrowth: roles of antibiotics, prebiotics, and probiotics. Gastroenterology 2006;130:S78-90.

[10] Dibaise JK, Young RJ, Vanderhoof JA. Enteric microbial flora, bacterial overgrowth, and shortbowel syndrome. Clin Gastroenterol Hepatol 2006;4:11-20.

[11] Hukkinen M, Kivisaari R, Merras-Salmio L, et al. Small bowel dilatation predicts prolonged parenteral nutrition and decreased survival in pediatric short bowel syndrome. Ann Surg 2016. http://dx.doi.org/10.1097/SLA.0000000000001893 [in press].

[12] Kaufman SS, Loseke CA, Lupo JV, et al. Influence of bacterial overgrowth and intestinal inflammation on duration of parenteral nutrition in children with short bowel syndrome. J Pediatr 1997;131:356-61.

[13] Gutierrez IM, Kang KH, Calvert CE, et al. Risk factors for small bowel bacterial overgrowth and diagnostic yield of duodenal aspirates in children with intestinal failure: a retrospective review. J Pediatr Surg 2012;47:1150-4.

[14] Jones BA, Hull MA, Potanos KM, et al. Report of 111 consecutive patients enrolled in the International Serial Transverse Enteroplasty (STEP) Data Registry: a retrospective observational study. J Am Coll Surg 2013;216:438-46.

[15] Oliveira C, de Silva N, Wales PW. Five-year outcomes after serial transverse enteroplasty in children with short bowel syndrome. J Pediatr Surg 2012;47:931-7.

[16] Almond SL, Haveliwala Z, Khalil B, et al. Autologous intestinal reconstructive surgery to reduce bowel dilatation improves intestinal adaptation in children with short bowel syndrome. J Pediatr Gastroenterol Nutr 2013;56:631-4.

[17] Iyer KR. Surgical management of short bowel syndrome. JPEN J Parenter Enteral Nutr 2014;38:S53-9.

[18] Mercer DF, Hobson BD, Gerhardt BK, et al. Serial transverse enteroplasty allows children with short bowel to wean from parenteral nutrition. J Pediatr 2014;164:93-8.

[19] Pakarinen MP. Autologous intestinal reconstruction surgery as part of comprehensive management of intestinal failure. Pediatr Surg Int 2015;31:453-64.

[20] Wester T, Borg H, Naji H, et al. Serial transverse enteroplasty to facilitate enteral autonomy in selected children with short bowel syndrome. Br J Surg 2014;101: 1329-33.

[21] Jones BA, Hull MA, McGuire MM, et al. Autologous intestinal reconstruction surgery. Semin Pediatr Surg 2010;19:59-67.

[22] Hukkinen M, Merras-Salmio L, Sipponen T, et al. Surgical rehabilitation of short and dysmotile intestine in children and adults. Scand J Gastroenterol 2015;50:153-61.

[23] Thompson JS, Quigley EM, Adrian TE. Effect of intestinal tapering and lengthening on intestinal structure and function. Am J Surg 1995;169:111-9.

[24] Reinshagen K, Zahn K, Buch C, et al. The impact of longitudinal intestinal lengthening and tailoring on liver function in short bowel syndrome. Eur J Pediatr Surg 2008;18: 249-53.

[25] Pakarinen MP, Kurvinen A, Koivusalo AI, et al. Long-term controlled outcomes after autologous intestinal reconstruction surgery in treatment of severe short bowel syndrome. J Pediatr Surg 2013;48:339-44.

[26] Merras-Salmio L, Pakarinen MP. Refined multidisciplinary protocol based approach to short bowel syndrome improves outcomes. J Pediatr Gastroenterol Nutr 2015: 61:24-9.

[27] Avitzur Y, Grant D. Intestine transplantation in children: update 2010. Pediatr Clin North Am 2010;57:415-31.

[28] Struijs MC, Diamond IR, de Silva N, et al. Establishing norms for intestinal length in children. J Pediatr Surg 2009;44:933-8.

[29] Kang KH-J, Gutierrez IM, Zurakowski D, et al. Bowel redilation following serial transverse enteroplasty (STEP). Pediatr Surg Int 2012;28:1189-93.

[30] Tander B, Bicakci U, Sullu Y, et al. Alterations of Cajal cells in patients with small bowel atresia. J Pediatr Surg 2010;45:724-8.

[31] Masumoto K, Suita S, Nada O, et al. Abnormalities of enteric neurons, intestinal pacemaker cells, and smooth muscle in human intestinal atresia. J Pediatr Surg 1999;34: 1463-8.

[32] Masumoto K, Suita S, Nada O, et al. The occurrence of unusual smooth muscle bundles expressing alpha-smooth muscle actin in human intestinal atresia. J Pediatr Surg 2003;38:161-6.

[33] Dicken BJ, Sergi C, Rescorla FJ, et al. Medical management of motility disorders in patients with intestinal failure: a focus on necrotizing enterocolitis, gastroschisis, and intestinal atresia. J Pediatr Surg 2011;46:1618-30.

[34] Srinathan SK, Langer JC, Blennerhassett MG, et al. Etiology of intestinal damage in gastroschisis. III: morphometric analysis of the smooth muscle and submucosa. J Pediatr Surg 1995;30:379-83.

[35] Krebs T, Boettcher M, Schafer H, et al. Gut inflammation and expression of ICC in a fetal lamb model of fetoscopic intervention for gastroschisis. Surg Endosc 2014;28:2437-42.

[36] Auber F, Danzer E, Noche-Monnery M, et al. Enteric nervous system impairment in gastroschisis. Eur J Pediatr Surg 2013;23:29-38. 
[37] Zani-Ruttenstock E, Zani A, Paul A, et al. Interstitial cells of Cajal are decreased in patients with gastroschisis associated intestinal dysmotility. J Pediatr Surg 2015;50: $750-4$

[38] Sala D, Chomto S, Hill S. Long-term outcomes of short bowel syndrome requiring longterm/home intravenous nutrition compared in children with gastroschisis and those with volvulus. Transplant Proc 2010;42:5-8.

[39] Khan FA, Squires RH, Litman HJ, et al. Predictors of enteral autonomy in children with intestinal failure: a multicenter cohort study. J Pediatr 2015;167:29-34e1.

[40] Spencer AU, Neaga A, West B, et al. Pediatric short bowel syndrome: redefining predictors of success. Ann Surg 2005;242:403-12.
[41] Sondheimer JM, Cadnapaphornchai M, Sontag M, et al. Predicting the duration of dependence on parenteral nutrition after neonatal intestinal resection. J Pediatr 1998; 132:80-4.

[42] Asensio AB, Garcia-Urkia N, Aldazabal P, et al. Incidence of bacterial translocation in four different models of experimental short bowel syndrome. Cir Pediatr 2003:16:20-5.

[43] Jeppesen PB. Modern treatment of short bowel syndrome. Curr Opin Clin Nutr Metab Care 2013;16:582-7.

[44] Modi BP, Javid PJ, Jaksic T, et al. First report of the international serial transverse enteroplasty data registry: indications, efficacy, and complications. J Am Coll Surg 2007;204:365-71. 